Marquette University

e-Publications@Marquette

9-1-2016

\title{
Muscle-Specific Effective Mechanical Advantage and Joint Impulse in Weightlifting
}

Kristof Kipp

Marquette University, kristof.kipp@marquette.edu

Chad Harris

LaGrange College

Accepted version. Journal of Strength and Conditioning Research (September 2016). DOI. (C) 2016 Lippincott Williams \& Wilkins, Inc. Used with permission. 


\title{
Muscle-Specific Effective Mechanical Advantage and J oint Impulse in Weightlifting
}

\author{
Kipp, Kristof \\ Department of Physical Therapy_Program in Exercise Science, Marquette University, \\ Milwaukee, WI \\ Harris, Chad \\ Department of Health Science, Central Oregon Community College, Bend, OR
}

\begin{abstract}
Kipp, K, and Harris, C. Muscle-specific effective mechanical advantage and joint impulse in weightlifting. J Strength Cond Res 31(7): 1905-1910, 2017-Lifting greater loads during weightlifting exercises may theoretically be achieved through increasing the magnitudes of net joint impulses or manipulating the joints' effective mechanical advantage (EMA). The purpose of this study was to investigate muscle-specific EMA and joint impulse as well as impulse-momentum characteristics of the lifter-barbell system across a range of external loads during the execution of the clean. Collegiate-level weightlifters performed submaximal cleans at 65,75 , and $85 \%$ of their 1-repetition maximum (1-RM), whereas data from a motion analysis system and a force plate were used to calculate lifter-barbell system impulse and velocity, as well as net extensor impulse generated at the hip, knee, and ankle joints and the EMA of the gluteus maximus, hamstrings, quadriceps, and triceps surae muscles. The results indicated that the lifter-barbell system impulse did not change as load increased, whereas the velocity of the lifter-barbell system decreased with greater load. In addition, the net extensor impulse at all joints increased as load increased. The EMA of all muscles did not, however, change as load increased. The load-dependent effects on the impulse-velocity characteristics of the lifter-barbell system may reflect musculoskeletal force-velocity behaviors, and may further indicate that the weightlifting performance is limited by the magnitude of ground reaction force impulse. In turn, the load-dependent effects observed at the joint level indicated that lifting greater loads were due to
\end{abstract}


NOT THE PUBLISHED VERSION; this is the author's final, peer-reviewed manuscript. The published version may be accessed by following the link in the citation at the bottom of the page.

greater net extensor impulses generated at the joints of the lower extremity and not greater EMAs of the respective extensor muscles. In combination, these results suggest that lifting greater external loads during the clean is due to the ability to generate large extensor joint impulses, rather than manipulate EMA.

Key Words: dynamic gearing; gear ratio; biomechanics

\section{Introduction}

Performance in the sport of weightlifting is dictated by the barbell mass a

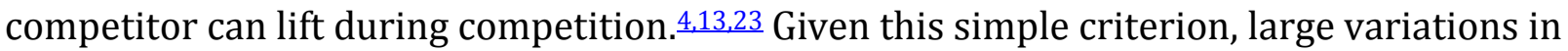
weightlifting technique are generally not observed among weightlifters as gross characteristics in technique are very similar across diverse experience or skill levels. $\frac{9.10}{9}$ For example, almost all weightlifters use a pulling technique that involves the so-called double knee bend, which is characterized by a very dynamic interaction and stereotypical extension-flexion-extension pattern of the lower extremity joints, especially the

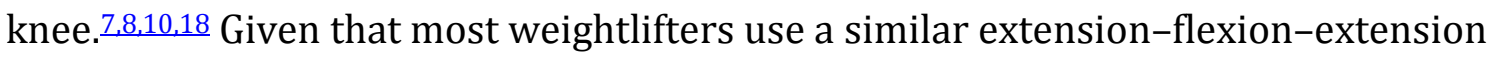
pattern as part of their technique, it is often posited that better lifters lift more weight because they are stronger, and are able to generate greater peak joint moments during the various pulling phases of either the snatch or clean.

Although maximal muscle strength is an important physical ability that influences performance in weightlifting, the significance of the ability to generate peak joint moments is less clear. $\underline{1,24}$ For example, it has been reported that the peak joint moments generated by the lower extremity joints during the clean do not always increase with changes in load across submaximal percentages. $\frac{15,17}{17}$ Furthermore, with respect to maximal loads, Baumann et al. (1988) reported that only peak hip extensor moments during the pull phase of the snatch correlated with the maximal load lifted during competition, whereas peak knee and ankle moments did not. However, after further examination of the dynamic time series data of the knee joint moment and the external moment arm of the ground reaction force about the knee joint, the authors suggested that better lifters were able to limit the peak knee joint moment by precise control of the knee position with respect to the ground reaction force vector. 1 Similarly, Enoka (1979) suggested that one of the key functions of the double-knee bend technique is to reposition the body so as to minimize external moment arms and minimize the necessary joint moments required to perform the second pull during the clean.

Although Baumann et al. (1988) and Enoka (1979) presented the idea that the control of the ground reaction force vector and the resultant external moment arm about a joint, is an important aspect of weightlifting technique; that idea was not based on empirical data as these authors did not actually quantify the mechanical advantage that is 
conferred by the control of the external moment arm. The effective mechanical advantage (EMA) of a muscle is often calculated as the ratio between the internal moment arm of a muscle at a specific joint and the external moment arm of the ground reaction force vector about that joint. $\underline{2,3}$ This ratio is also sometimes referred to as the gear ratio, because changing the mechanical advantage allows muscles and joints to operate like a dynamic gear in that it provides a mechanism to effectively transfer force or velocity between 2 structures and "shift" more effectively between high force and low velocity and low force and high velocity scenarios. $\underline{2,3,14}$ The EMA is influenced by changes in limb posture with respect to the ground reaction force vector and affects the leverage of muscles. $\underline{2}$ Changes in the EMA can thus directly affect the muscular effort required to effectively translate muscle force into movement. $\frac{2,3}{3}$ In the case of weightlifting, manipulating the EMA may therefore hypothetically enable a lifter to lift heavier loads and mitigate the need for greater muscle forces or concomitant net joint moments during the pull phases of weightlifting exercises.

Although the EMA concept may help explain the lack of association between peak joint moments and performance at either submaximal or maximal loads, it should be noted that the EMA during dynamic movement is directly related to the mechanical joint impulse that is generated or required for the successful execution of said movement. $\underline{2}$ It would therefore seem important to also study the impulse-momentum characteristics of the lower extremity joints in addition to the EMA of the lower extremity muscles during weightlifting movements. Moreover, the impulse-momentum characteristics of the lifterbarbell system should also be considered, because lifting heavier loads may not necessarily require a greater vertical ground reaction force impulse if the load is lifted at a lower velocity. The trade-off between load and velocity could thus hypothetically mitigate the need to change the required muscle effort at all.

Based on the aforementioned lack of knowledge about the role of muscle-specific EMA and joint impulse, as well as impulse-momentum characteristics of the lifter-barbell system, in relation to weightlifting performance the purpose of this study was to systematically investigate these variables during a weightlifting exercise (i.e., the clean) across a range of submaximal loads. We hypothesized that weightlifters change the EMA of their lower extremity muscles during the clean such that an increase in load would also be accompanied by a favorable change in the EMA of the lower extremity muscles, but not necessarily in the joint impulse. The confirmation of this hypothesis would offer a deeper insight into the mechanisms that drive performance in the sport of weightlifting and provide coaches and sports scientists with applied information about technical factors that could be improved and monitored during the training of weightlifters. 
NOT THE PUBLISHED VERSION; this is the author's final, peer-reviewed manuscript. The published version may be accessed by following the link in the citation at the bottom of the page.

\section{Methods}

\section{Experimental Approach to the Problem}

Data collection occurred after all subjects performed a self-paced warm-up that included lifting lighter loads between 35 and 50\% of their 1-Repetition Maximum (RM) for the clean exercise. After the warm-up, subjects performed 3 repetitions each at 65 and $75 \%$ of $1-\mathrm{RM}$, performed 2 repetitions at $85 \%$ of $1-\mathrm{RM}$, and were given $2-3$ minutes rest between each set. All kinematic and kinetic data were collected during each of the 3 target sets (i.e., 65,75 , and $85 \%$ of 1-RM). Data from within each set were averaged and used for statistical comparisons.

\section{Subjects}

We recruited 6 male subjects to participate in this study (Mean \pm SD; age: $24.8 \pm$ 0.8; height: $1.85 \pm 0.09 \mathrm{~m}$; mass: $106.0 \pm 13.2 \mathrm{~kg}$; absolute $1-\mathrm{RM}$ clean: $126.4 \pm 22.9 \mathrm{~kg}$; relative 1-RM clean: $\left.1.19 \pm 0.11 \mathrm{~kg} \cdot \mathrm{kg}^{-1}\right)$. Subjects self-reported their $1-\mathrm{RMs}$, which were and current within 2 weeks of testing. All subjects were deemed technically competent and representative of collegiate-level weightlifters by a national USA Weightlifting coach. All subjects provided written informed consent after reading an informed consent document approved by the University's Institutional Review Board.

\section{Procedures}

\section{Data Collection}

A motion analysis system (Vicon 612; Vicon, Los Angeles, CA, USA) was used to record the 3-dimensional position of reflective markers at $250 \mathrm{~Hz}$. Markers were attached bilaterally to the anterior and posterior superior iliac spines, the lateral and medial epicondyles, the lateral and medial malleoli, and the heel and toe of each foot.17 Two force plates (Type 9281E; Kistler Instrument Corp., Amherst, NY, USA), which were built into a $2.44 \times 2.44 \times 0.10 \mathrm{~m}$ weightlifting platform, were simultaneously used to record kinetic data from both feet at $1,250 \mathrm{~Hz}$.

\section{Data Processing}

Kinematic and kinetic data were low-pass filtered at 6 and $25 \mathrm{~Hz}$, respectively. To calculate lifter-barbell system impulse and velocity, the vertical ground reaction force vectors from each of the force plates were first algebraically summed into a single ground reaction force vector. The vertical impulse was calculated through numerical integration (i.e., use of the trapezoidal rule) of the net vertical ground reaction force, which was defined as the difference between total vertical ground reaction force and the force of gravity acting on the center of mass of the lifter-barbell system. The velocity of the lifter- 
barbell system was calculated through numerical integration (i.e., use of the trapezoidal rule) of the acceleration of the center of mass of the lifter-barbell system, which was defined as the difference between the ratio of the total vertical ground reaction force and lifter-barbell system mass and the gravity constant (i.e., $9.81 \mathrm{~m} \cdot \mathrm{s}^{-2}$ ). 16 The net vertical ground reaction force impulse (System Impulse) and the peak velocity (System Velocity) of the lifter-barbell system were then extracted and used for analysis.

Euler angle rotation sequences were used to calculate ankle, knee, and hip joint angles. Kinematic and kinetic data then were combined with published anthropometric data 5 and used to solve for ankle, knee, and hip joint moments with an inverse dynamics approach. $\underline{25}$ Calculated joint moments represent net internal moments and were normalized to body-mass and height. For ease of comparison, all joint moments timeseries data were processed such that a positive moment represented a net internal extensor moment. Net extensor joint impulse (Joint Impulse) was calculated by numerically integrating the normalized joint moment time-series data with respect to time whenever a positive extensor moment was present. 25 All kinematic and kinetic time-series data were trimmed from the time the barbell broke contact with the platform to the time the vertical ground reaction force fell below $10 \mathrm{~N}$. This time frame therefore captured the first and second pull phase of the clean, along with the transition between these phases.

The external moment arms about each joint were calculated as the perpendicular distance between the vector of the vertical and horizontal ground reaction forces and each respective lower extremity joint center. $\underline{3}$ Figure 1 depicts the time-varying characteristics of the ground reaction force vector about the lower extremity joint centers. The internal moment arms of the major extensor muscles of the ankle (triceps surae), knee (quadriceps), and hip (gluteus maximum and hamstrings) joints were calculated with regression equations that were calculated from previously established

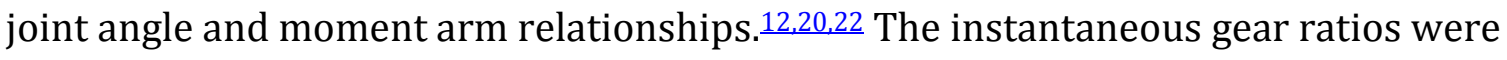
calculated as the ratio between the internal moment arm of the respective muscles about the joint they cross and the external moment arm of the ground reaction force vector about that joint. $\frac{14}{1}$ Gear ratio data were averaged over the course of the pull phase to obtain the average effective mechanical advantage (EMA) of the gluteus maximus, hamstring, quadriceps, and triceps surae muscle groups during each lift. Pilot testing indicated that kinematic and kinetic data exhibited high reliability (intraclass correlation coefficients $>0.90$ ).

\section{Statistical Analyses}

The statistical analysis of the lifter-barbell system data included 2 dependent variables (i.e., System Impulse and System Velocity) and one independent variable (i.e., 
Load). Two separate analyses of variance (ANOVA) with repeated measures (i.e., one for each dependent variable) were used to investigate differences among the different levels of the independent variable. The statistical analysis of the muscle- and joint-specific data included 2 dependent variables (i.e., EMA and Joint Impulse) and 2 independent variables (i.e., Load and Muscle or Joint). Two separate ANOVAs with repeated measures (i.e., one for each dependent variable) were used to investigate differences among independent variables. In each ANOVA, load was treated as a repeated measure. Mauchly's test of Sphericity was used to assess whether the data met the statistical assumptions of the test statistic. Greenhouse-Geiser corrections were made when appropriate. A priori alphalevels for statistical significance were set at 0.05. Post hoc comparisons were made with ttests. Data are presented as Mean \pm Standard Deviation (SD). All statistical analyses were performed in SPSS 22 (IBM, New York, NY, USA).

Results

\section{System Impulse and Velocity}

The preliminary statistical analysis for the system impulse and velocity showed that assumptions of sphericity were met. The ANOVA results showed a significant main effect of load on system velocity $\left(p=0.035\right.$, $[\text { eta }]^{2}=0.488$, power $\left.=0.656\right)$, but not system impulse $\left(p=0.214\right.$, $[\text { eta }]^{2}=0.266$, power $\left.=0.291\right)$ ( $\left.\underline{\text { Table } 1}\right)$. Within-subject contrasts showed that system velocity exhibited a linear trend $\left(p=0.077\right.$, $[\mathrm{eta}]^{2}=0.498$, power $=0.437)$ towards lower velocities at higher loads. The post hoc tests of the pooled estimated marginal means indicated that this trend was the result of a statistically significant difference $(p=0.037)$ in system velocity between loads at 75 and $85 \%$ of 1 RM.

\section{EMA and Joint Impulse}

The preliminary statistical analysis for the EMA showed that assumptions of sphericity were not met; the Greenhouse-Geiser correction was therefore used to adjust the significance level. The ANOVA results showed no significant interactions between load and muscle on EMA $\left(p=0.881\right.$, $[\text { eta }]^{2}=0.046$, power $\left.=0.116\right)$ (Table 2). Significant main effects on EMA existed only for muscle $\left(p=0.001\right.$, $[\text { eta }]^{2}=0.683$, power $\left.=1.000\right)$, but not for load $\left(\mathrm{p}=0.236\right.$, $[\mathrm{eta}]^{2}=0.070$, power $\left.=0.262\right)$. The post hoc analysis of the pooled estimated marginal means for EMA indicated significant differences between the gluteus maximus and triceps surae $(p=0.001)$, the gluteus maximus and quadriceps $(p=0.001)$ muscles, as well as between the hamstrings and triceps surae $(p=0.016)$, and the hamstrings and quadriceps $(\mathrm{p}=0.021)$ muscles. 
The preliminary statistical analysis for net joint impulse showed that assumptions of sphericity were met. The ANOVA showed a significant main effect for load $(\mathrm{p}=0.001$, $[\text { eta }]^{2}=0.401$, power $\left.=0.975\right)$ and joint $\left(p=0.001\right.$, $[\text { eta }]^{2}=0.760$, power $\left.=1.000\right)$, but there were no significant interactions between the 2 variables $(p=0.163 \text {, [eta }]^{2}=0.190$, power $=0.472)(\underline{\text { Table } 3})$. Within-subject contrasts indicated a linear trend for joint impulse $\left(p=0.001\right.$, $[\text { eta }]^{2}=0.553$, power $\left.=0.980\right)$ across loads, and post hoc analysis of the pooled estimated marginal means for joint impulse indicated significant increases from 65 to $75 \%(\mathrm{p}=0.029)$ and from 65 to $85 \%(\mathrm{p}=0.002)$ loads. In addition, post hoc analysis of the pooled estimated marginal means for joint impulse indicated significant differences between the hip and ankle $(\mathrm{p}=0.001)$ and between the hip and knee $(\mathrm{p}=$ $0.001)$ joint.

\section{Discussion}

The purpose of this study was to investigate muscle-specific EMA and joint impulse as well as impulse-momentum characteristics of the lifter-barbell system across a range of external loads during the execution of the clean. We hypothesized that an increase in external load would occur because of concomitant improvements in the mechanical advantage of lower extremity muscles, but not necessarily increases in the mechanical impulse generated at the lower extremity joints. Contrary to this hypothesis, however, the results indicated that as load increased the net extensor impulse at all joints also increased, but the EMA of all muscles did not. In addition, the lifter-barbell system impulse did not change as load increased, whereas the velocity of the lifter-barbell system decreased with greater load. In combination, these results highlight the importance of the ability to generate large extensor muscle joint impulses, rather than manipulate muscle EMA, in an effort to lift greater external loads during the clean.

Several researchers have proposed that the manipulation of the external moment arm about a joint mitigates the need to generate large peak joint moments, and thus enables weightlifters to lift heavy loads during the pull phases of weightlifting exercises without the need for equally large muscle efforts. $\frac{1,6}{}$ This proposal is intriguing because it would help clarify the role of joint kinetics in relation to weightlifting performance, and partially explain the lack of correlation between peak joint moments and the lifted loads.1.15 In light of this assertion, we hypothesized that weightlifters would manipulate the EMA of their lower extremity muscles as they lifted increasingly larger external loads over the course of several submaximal sets of the clean exercise. The results of this study, however, indicated that weightlifters did not alter the EMA as external load increased through the range of $65-85 \%$ of 1 -RM. This finding therefore indicates that EMA does not exhibit load-dependent behavior through a range of submaximal efforts. In contrast, 
Baumann et al. (1988) illustrated that the gold medal winner at the 1985 world weightlifting championships exhibited better EMA than a lifter who finished much lower in the same weight category at the same competition. Interestingly, the better lifter exhibited a smaller peak knee joint moment, which would suggest that the greater load lifted by the gold medalist was partially associated with an increase in the efficiency of lower extremity biomechanics, because a better EMA would allow the lifter to generate greater ground reaction forces without a concomitant and necessary increase in the muscular effort of the quadricep muscles. 1 The discrepancy between these findings may be due to any number of reasons; likely reasons may be the experimental design and calculation of EMA. In the current study, we studied changes in EMA within the same lifters but across submaximal loads, whereas Baumann et al. ${ }^{1}$ presented data on EMA between different lifters. In addition, Baumann et al. $\underline{1}$ didn't quantify or statistically compare EMA in their study. Regardless, it should be noted that this does not mean that weightlifters don't change or dynamically "gear" the EMA of their muscles during the different pull phases of weightlifting exercises so as to minimize muscle effort at specific joints, which is what others have observed during sprinting and running. $\underline{2,14}$ Arguably, weightlifters are certainly expected to change the EMA of several large extensor muscles during selected phases of the clean or snatch because they reposition their bodies with respect to the barbell, as observed during the double-knee bend technique.1.6-11 Since we averaged EMA across all pull phases in the current study, it is conceivable that EMA does actually differ between pull phases and could thus also be affected by load, but possibly only during selected pull phases. Whether EMA should be considered an important aspect of weightlifting technique is therefore still to be determined.

The mechanical impulse generated at the hip, knee, and ankle joints increased linearly from 65 to $75 \%$ and from 65 to $85 \%$ of 1-RM. Although no studies have previously examined load-dependent changes in joint impulse during weightlifting exercises, other previous investigations reported that discrete peak values of joint moment and powers do not necessarily increase linearly as load increases across similar submaximal ranges. .115 For example, Kipp et al. $\underline{15}$ reported that an increase in load across submaximal percentages is not always associated with a concomitant increase in peak hip, knee, and ankle joint moments. Given the linear trends in the statistical analysis of the effects of load on net joint impulse for all 3 joints, it thus seems that the ability to generate large joint impulses, rather than large peak joint moments, is an important prerequisite to lift heavier external loads. In addition, the joint-dependent differences in mechanical impulse noted in the current study, specifically the significantly larger joint impulse observed at the hip further underscore the importance of the hip joint function and strength in relation to weightlifting performance. 18 This joint-specific difference in mechanical impulse may also 
help explain why in a previous study, only the peak hip extensor moment and not the peak knee and ankle moments, during the pull phase of the snatch correlated with maximal lift weight during competition. 1 Time-series analysis of joint moment data generally shows that the hip generates a large, constant moment during all pull phases, whereas the knee and ankle joints exhibit a much more dynamic extension-flexion-extension pattern. $\frac{17,18}{\text { In }}$ light of the dynamic nature of weightlifting exercises, the patterning of knee and ankle moments may point to a greater role for large rates of force development than large peak force production in relation to weightlifting performance. $\frac{11,15}{} \mathrm{~A}$ similar case has been made for other fast and dynamic movements such as vertical jumping. $\underline{19}$ which weightlifting exercises are often compared with. $.4,21$

With respect to the impulse-momentum characteristics of the lifter-barbell system, the results indicated that the system impulse did not change as load increased, whereas the velocity of the lifter-barbell system decreased with greater load. These load-dependent effects likely reflect the expression of musculoskeletal force-velocity behaviors in that the velocity of the lifter-barbell system decreases as its load increases. $\underline{25}$ Given that the net impulse of the vertical ground reaction force acting on the lifter-barbell system remained constant across loads may make it attractive to speculate that the impulse's magnitude is a primary limiter of weightlifting performance. Hypothetically, larger ground reaction force impulses could be used to increase the external load-even in the face of decreasing system velocity, which would eventually reach a critical minimum needed to project the system, and in particular the barbell, upwards and into the support position-once the critical minimum is reached any remaining "excess" impulse could be used to increase the load. The veracity of such speculation still needs to be more rigorously examined in future studies. In addition, the lack of agreement between joint-level and system-level effects with regards to load-dependent changes in impulse warrants a brief discussion because one may suppose that increases in joint impulse would directly translate into increases in system impulse. For one, there may be other joints and muscles that contribute to total system impulse that were not considered in our analysis. Specifically, the biomechanical model used in the current study used a rigid-link segment model to analyze the hip, knee, and ankle joints but does not provide information about the biomechanics of the trunk and upper extremity joints.

The results and conclusions of this study should be interpreted in light of one additional limitation not discussed above. In this study, we only calculated the "net" internal joint moments, which implies that only the net sum of all joint moments that act about a joint is considered and that the effects of coactivation from antagonistic muscle group are ignored. $\underline{25}$ Hence, a net joint "extension" moment only indicates that the moment created by the "extensor" muscles is greater than that of the flexor muscles, but 
also implies that the actual muscle forces that create this moment could in reality be much larger. This limitation could thus lead to an under-estimation of the actual muscle forces and possibly joint impulse, which in turn may affect the EMA calculations. Although this may not be a problem for the knee and ankle joints, because the shared tendinous insertion points of the quadriceps and triceps surae muscle groups provide a discrete moment arm of these extensor muscles, it may be so for the hip because the major extensor muscles that act across this joint do not have similar moment arms. $\stackrel{22}{\text { Our }}$ musculoskeletal model and calculation of EMA did not account for possible differences in load-sharing strategies between the major extensor muscles of the hip joint. We addressed this issue in part by calculating the EMA of the hamstrings and gluteus maximus separately.

\section{Practical Applications}

The results of the current study suggest that lifting greater external loads during the clean is due to the ability to generate larger mechanical impulses at the hip, knee, and ankle joints rather than the manipulation of the mechanical advantage of the extensor muscles at these joints. In addition, the results suggest that the hip joint generated significantly larger joint impulse than the ankle and knee joint. When interpreted in light of other literature, these results likely highlight the importance of the ability to generate large extension impulses at the joints of the lower extremities, especially at the hip, in relation to load-dependent changes in weightlifting biomechanics and performance.

\section{References}

${ }^{1}$ Baumann W, Gross V, Quade K, Galbierz P, Schwirtz A. The snatch technique of world class weightlifters at the 1985 world championships. Int J Sport Biomech 4: 68-89, 1988.

${ }^{2}$ Biewener AA, Farley CT, Roberts TJ, Temaner M. Muscle mechanical advantage of human walking and running: Implications for energy cost. J App Physiol 97: 2266-2274, 2004.

${ }^{3}$ Carrier DR, Heglund NC, Earls KD. Variable gearing during locomotion in the human musculoskeletal system. Science 265: 651-653, 1994.

${ }^{4}$ Chiu LZ, Schilling BA. Primer on weightlifting: From sport to sports training. Strength Cond J27: 42$48,2005$.

5DeLeva P. Adjustments to Zatsiorsky-Seluyanov's segment inertia parameters. J Biomech 29: 1223$1230,1996$.

${ }^{6}$ Enoka RM. The pull in Olympic weightlifting. Med Sci Sports 11: 131-137, 1979.

${ }^{7}$ Enoka RM. Load- and skill-related changes in segmental contributions to a weightlifting movement. Med Sci Sports Exerc 20: 178-187, 1988.

Journal of Strength and Conditioning Research, Vol. 31, No. 7 (July 2017): pg. 1905-1910. DOI. This article is @ Lippincott Williams \& Wilkins, Inc. and permission has been granted for this version to appear in e-Publications@Marquette. Lippincott Williams \& Wilkins, Inc. does not grant permission for this article to be further copied/distributed or hosted elsewhere without the express permission from Lippincott Williams \& Wilkins, Inc. 
NOT THE PUBLISHED VERSION; this is the author's final, peer-reviewed manuscript. The published version may be accessed by following the link in the citation at the bottom of the page.

${ }^{8}$ Garhammer J. Biomechanical characteristics of the 1978 world weightlifting champions. Int Series Biomech 3: 300-304, 1981.

${ }^{9}$ Garhammer J. Biomechanical profiles of olympic weightlifters. Int J Sport Biomech 1: 122-130, 1985.

${ }^{10}$ Gourgoulis V, Aggeloussis N, Kalivas V, Antoniou P, Mavromatis G. Snatch lift kinematics and bar energetics in male adolescent and adult weightlifters. J Sports Med Phys Fitness 44: 126-131, 2004.

${ }^{11}$ Hadi G, Akkus H, Harbili E. Three-dimensional kinematic analysis of the snatch technique for lifting different barbell weights. J Strength Cond Res 26: 1568-1576, 2012.

${ }^{12}$ Herzog W, Read LJ. Lines of action and moment arms of the major force-carrying structures crossing the human knee joint. J Anat 182: 213-230, 1993.

${ }^{13}$ Ho LKW, Lorenzen C, Wilson CJ. Reviewing current knowledge in snatch performance and technique: the need for future directions in applied research. J Strength Cond Res 28: 574-586, 2014.

${ }^{14}$ Karamanidis K, Arampatzis A. Aging and running experience affects the gearing in the musculoskeletal system of the lower extremities while walking. Gait Posture 25: 590-596, 2007.

${ }^{15}$ Kipp K, Harris C, Sabick MB. Lower extremity biomechanics during weightlifting exercise vary across joint and load. J Strength Cond Res 25: 1229-1234, 2011.

${ }^{16}$ Kipp K, Harris C, Sabick MB. Correlations between internal and external power outputs during weightlifting exercise. J Strength Cond Res 27: 1025-1030, 2013.

${ }^{17}$ Kipp K, Redden J, Sabick MB, Harris C. Kinematic and kinetic synergies of the lower extremities during the pull in olympic weightlifting. J App Biomech 26: 271-278, 2012a.

${ }^{18} \mathrm{Kipp} \mathrm{K}$, Redden J, Sabick MB, Harris C. Weightlifting performance is related to kinematic and kinetic patterns of the hip and knee joints. J Strength Cond Res 26: 1838-1844, 2012b.

${ }^{19}$ Laffaye G, Wagner PP, Tombleson TIL. Countermovement jump height: Gender and sport-specific differences in the force-time variables. J Strength Cond Res 28: 1096-1105, 2014.

${ }^{20}$ Maganaris CN, Baltzopoulos V, Sargeant AJ. Changes in achilles tendon moment arm from rest to maximum isometric plantarflexion: In vivo observations in man. J Physiol510: 977-985, 1998.

${ }^{21}$ Moolyk AN, Carey JP, Chiu ZF. Characteristics of lower extremity work during the impact phase of jumping and weightlifting. J Strength Cond Res 27: 3225-3232, 2013.

${ }^{22}$ Németh G, Ohlsén H. In vivo moment arm lengths for hip extensor muscles at different angles of hip flexion. J Biomech 18: 129-140, 1985.

23Stone MH, Pierce KC, Sands WA, Stone ME. Weightlifting: A brief overview. Strength Cond J28: 50-66, 2006.

${ }^{24}$ Stone MH, Sands WA, Pierce KC, Carlock J, Cardinale M, Newton RU. Relationship of maximum strength to weightlifting performance. Med Sci Sports Exerc 37: 1037-1043, 2005.

25Winters DA. Biomechanics and Motor Control of Human Movement. Hoboken, NJ: John Wiley \& Sons, 2003.

Journal of Strength and Conditioning Research, Vol. 31, No. 7 (July 2017): pg. 1905-1910. DOI. This article is (C) Lippincott Williams \& Wilkins, Inc. and permission has been granted for this version to appear in e-Publications@Marquette. Lippincott Williams \& Wilkins, Inc. does not grant permission for this article to be further copied/distributed or hosted elsewhere without the express permission from Lippincott Williams \& Wilkins, Inc. 

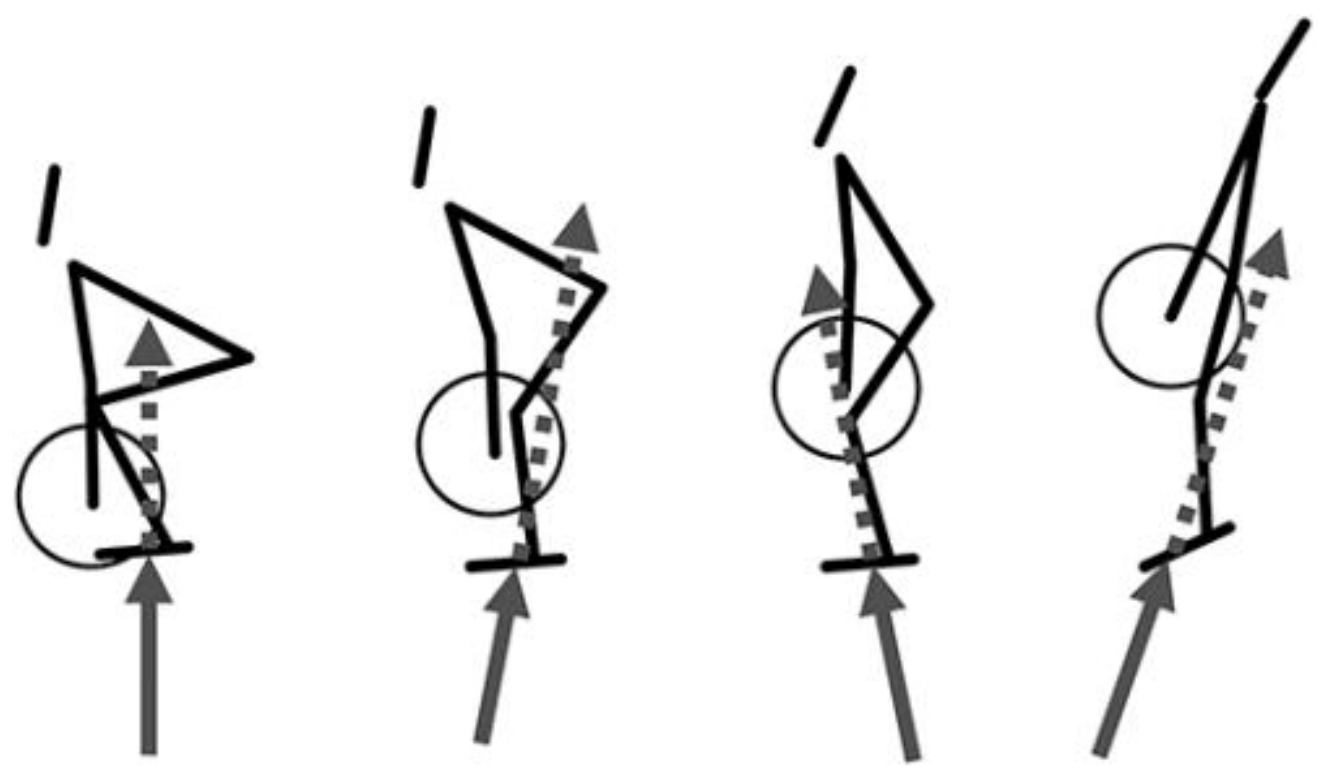

Figure 1. Projection of the ground reaction force vector (dotted lines) about the joints of the hip, knee, and ankle joints during the pull phase of the clean.

Journal of Strength and Conditioning Research, Vol. 31, No. 7 (July 2017): pg. 1905-1910. DOI. This article is @ Lippincott Williams \& Wilkins, Inc. and permission has been granted for this version to appear in e-Publications@Marquette. Lippincott Williams \& Wilkins, Inc. does not grant permission for this article to be further copied/distributed or hosted elsewhere without the express permission from Lippincott Williams \& Wilkins, Inc. 
NOT THE PUBLISHED VERSION; this is the author's final, peer-reviewed manuscript. The published version may be accessed by following the link in the citation at the bottom of the page.

\begin{tabular}{llc}
\hline Load & System impulse & System velocity \\
\hline 65 & $221.6 \pm 85.8$ & $1.21 \pm 0.32$ \\
75 & $240.9 \pm 96.0$ & $1.20 \pm 0.31$ \\
85 & $210.1 \pm 114.9$ & $1.02 \pm 0.34^{\star}$ \\
\hline
\end{tabular}

$\cdot p \leq 0.05$ vs. 75 .

Table 1 . Mean \pm SD system impulse $(\mathrm{N} \cdot \mathrm{s})$ and velocity $\left(\mathrm{m} \cdot \mathrm{s}^{-1}\right)$ of the lifter-barbell system center-of-mass at each load (\% of $1 \mathrm{RM})$.

\begin{tabular}{lcc}
\hline Muscle & Load & EMA \\
\hline Gluteus maximus & 65 & $3.87 \pm 1.11$ \\
& 75 & $3.81 \pm 1.21$ \\
Hamstring & 85 & $3.69 \pm 1.36$ \\
& 65 & $2.98 \pm 0.68$ \\
Quadriceps & 75 & $2.93 \pm 0.80$ \\
& 85 & $2.86 \pm 0.87$ \\
Triceps surae & 65 & $1.57 \pm 0.26^{\star} \dagger$ \\
& 75 & $1.57 \pm 0.20^{\star} \dagger$ \\
& 85 & $1.44 \pm 0.17^{\star} \dagger$ \\
& 65 & $1.45 \pm 0.23^{\star} \dagger$ \\
\hline
\end{tabular}

$\cdot p \leq 0.05$ vs. gluteus maximum.

$\div p \leq 0.05$ vs. hamstring.

Table 2. Mean \pm SD effective mechanical advantage (EMA [unitless]) for the lower extremity muscles at each load (\% of $1 \mathrm{RM})$.

\begin{tabular}{lcc}
\hline Joint & Load & Joint impulse \\
\hline Hip & 65 & $7.43 \pm 2.31$ \\
& 75 & $8.44 \pm 2.28^{\star}$ \\
Knee & 85 & $9.02 \pm 2.93^{\star}$ \\
& 65 & $2.21 \pm 0.73^{\star}$ \\
Ankle & 75 & $2.53 \pm 0.71^{\star} \dagger$ \\
& 85 & $2.46 \pm 0.53^{\star} \dagger$ \\
& 65 & $3.41 \pm 1.12^{\dagger}$ \\
& 75 & $4.00 \pm 1.23^{\star} \dagger$ \\
\hline
\end{tabular}

$* p \leq 0.05$ vs. 65 .

$\uparrow p \leq 0.05$ vs. hip.

Table 3. Mean \pm SD net mechanical joint impulse (joint impulse $\left[\mathrm{N} \cdot \mathrm{s} \cdot \mathrm{kg}^{-1}\right]$ ) for each lower extremity joint at each load (\% of $1 \mathrm{RM})$.

Journal of Strength and Conditioning Research, Vol. 31, No. 7 (July 2017): pg. 1905-1910. DOI. This article is @ Lippincott Williams \& Wilkins, Inc. and permission has been granted for this version to appear in e-Publications@Marquette. Lippincott Williams \& Wilkins, Inc. does not grant permission for this article to be further copied/distributed or hosted elsewhere without the express permission from Lippincott Williams \& Wilkins, Inc. 